\title{
Lidocaine liposome modified with folic acid suppresses the proliferation and motility of glioma cells via targeting the PI3K/AKT pathway
}

\author{
DEDONG LI ${ }^{1 *}$, XUEWEI YANG ${ }^{2 *}$, BO LI ${ }^{1}$, CHENYI YANG $^{3}$, JIAN SUN $^{1}$, \\ MINGDONG YU ${ }^{1}$, HAIYUN WANG ${ }^{3}$ and YUECHUN LU ${ }^{1}$ \\ ${ }^{1}$ Department of Anesthesiology, Second Hospital of Tianjin Medical University, Tianjin 300211; \\ ${ }^{2}$ Department of Anesthesiology, Tianjin Union Medical Center, Tianjin 300191; ${ }^{3}$ Department of \\ Anesthesiology, Tianjin Third Central Hospital, Tianjin 300052, P.R. China
}

Received September 4, 2020; Accepted May 28, 2021

DOI: 10.3892/etm.2021.10457

\begin{abstract}
Glioma is life-threatening tumor of the central nervous system. Although lidocaine is usually used as local anesthetic, it also has antitumor effects. However, its clinical application in glioma is hampered by limited distribution to the brain. The aim of the present study was to enhance the ability of lidocaine to penetrate the blood-brain barrier (BBB) to target glioma and investigate its antitumor mechanism. A folic acid (FA)-modified lidocaine-carrying liposome (Lid-FA-Lip) was prepared, and its particle size, $\zeta$ potential, encapsulation efficiency, release profile stability and hemolytic effect were characterized in vitro. The targeting capacity and antitumor activities of Lid-FA-Lip were also investigated in vitro and in vivo. The results indicated that the modification of liposomes with FA significantly improved the ability of lidocaine to cross the BBB in an in vitro model and increased its uptake by U87 cells. Additionally, Lid-FA-Lip significantly suppressed the motility of U87 glioma cells and stimulated apoptosis. Furthermore, the results confirmed that Lid-FA-Lip targeted the PI3K/AKT pathway and suppressed the growth of glioma xenografts in mice. In summary, the study demonstrated that Lid-FA-Lip is a promising liposomal formulation of lidocaine that may provide improved therapeutic effects on glioma, mediated via the PI3K/AKT pathway.
\end{abstract}

Correspondence to: Professor Yuechun Lu, Department of Anesthesiology, Second Hospital of Tianjin Medical University, 23 Pingjiang Road, Tianjin 300211, P.R. China

E-mail: luyuechunmzk@126.com

${ }^{*}$ Contributed equally

Key words: lidocaine, glioma, folic acid, PI3K/AKT pathway, proliferation

\section{Introduction}

Glioma is one of the most prevalent tumors of the central nervous system, with an age-adjusted ( $<78$ years old) annual incidence of 7.3 cases $/ 100,000$ individuals $(1,2)$. It is increasing in incidence by $\sim 1.2 \%$ each year (3). Notably, although treatment regimens comprising a combination of surgery and chemoradiotherapy are used to treat glioma $(4,5)$, the prognosis of patients with malignant glioma remains poor. The complete surgical removal of glioma is challenging as the tumor grows within the brain and often infiltrates the surrounding normal tissue $(6,7)$. Targeted therapy has become an important therapeutic method for the treatment of glioma $(8,9)$. Various targeted drug delivery systems, including liposomes and nano-micelles, have been used clinically or in clinical trials $(10,11)$. However, it is important to develop more effective drugs and delivery systems to improve the treatment of glioma and its outcomes.

Certain anesthetics have been found to have antitumor activities $(12,13)$, with effects on the apoptosis, proliferation, motility and drug resistance of various cancer cells. For example, one study demonstrated that clinically relevant concentrations of bupivacaine induced the apoptosis of sarcoma cells in vitro and suppressed their proliferation (14), and another study revealed that dibucaine had a pro-apoptotic effect on HL-60 promyelocytic leukemia cells (15). However, few studies have focused on the potential antitumor effects of lidocaine in glioma treatment.

The vascular-corrected concentration of drugs in the brain can be increased by using a higher drug dose; however, this may cause serious side effects and severe toxicity. Therefore, the development of effective systems to deliver anesthetics, such as lidocaine, into the brain is a topic of great interest. The targeted delivery of drugs through the blood-brain barrier (BBB) into the brain may not only increase treatment efficacy, but also enable the dose of drug administered to be reduced. Previous studies have focused on increasing the penetration of the BBB to improve glioma-targeting ability using specific ligands $(16,17)$. Folic acid (FA) is a most promising ligand for targeting the folate receptor (FR), which is overexpressed on 
the cell surface of multiple types of cancer cells, including U87 and MDA-MB-231 cells $(18,19)$. Additionally, the expression of the FR on normal cells is limited, which make it an attractive focus for efficient glioma-targeting. In addition, the FR is also highly expressed by cerebral capillary endothelial cells (19). Therefore, FA may have superior ability to cross the $\mathrm{BBB}$ and efficiently target glioma.

To verify the hypothesized ability of FA to cross the $\mathrm{BBB}$ and investigate the antitumor mechanisms of lidocaine, an innovative lidocaine-loaded liposome modified with FA (Lid-FA-Lip) was developed in the present study. This modified liposome was designed based on the assumption that FA will markedly increase the ability of the liposome to penetrate the BBB and thereby improve the antitumor effect. Whether Lid-FA-Lip can suppress the motility of glioma cells and stimulate apoptosis was also investigated. In addition, the antitumor effects of Lid-FA-Lip on the tumor growth of glioma cells in mice and the contribution of PI3K/AKT pathway suppression to the underlying mechanism were analyzed.

\section{Materials and methods}

\section{Chemistry}

Synthesis of cholesterol tosylate (compound 2). A solution of cholesterol (compound 1; $1.00 \mathrm{~g}, 2.59 \mathrm{mmol}$ ) in pyridine $(5 \mathrm{ml})$ was prepared, and a solution of $p$-toluenesulfonyl chloride $(0.74 \mathrm{~g}, 3.88 \mathrm{mmol})$ in pyridine $(5 \mathrm{ml})$ was added. After stirring the mixture for $10 \mathrm{~h}$ at $50^{\circ} \mathrm{C}$, the solvent was removed and the residue was re-dissolved with ethyl acetate $(20 \mathrm{ml})$. The solution was washed with $1 \mathrm{~mol} / 1 \mathrm{HCl}$ and saturated $\mathrm{NaCl}$. The solvent was then removed to yield compound 2 (1.19 g, 85\%), which was used for the next step without further purification. Melting point, $127-129^{\circ} \mathrm{C}$. High-resolution mass spectrometry (HRMS): Electrospray ionization $\left(\mathrm{ESI}^{+}\right)$calculated for $\mathrm{C}_{34} \mathrm{H}_{52} \mathrm{O}_{3} \mathrm{~S} \mathrm{Na}[\mathrm{M}+\mathrm{Na}]^{+}, 563.3535$; found, 563.3532. Elemental analysis: Calculated, C, 75.51; H, 9.69; S, 5.93; found, C, 75.43; H, 9.78; S, 6.11.

Synthesis of octaethylene glycol monocholesteryl ether (compound 3). To a solution of compound 2 (1.00 g, $1.85 \mathrm{mmol})$ in dioxane $(15 \mathrm{ml})$ was added octaethylene glycol $(6.85 \mathrm{~g}$, $18.49 \mathrm{mmol})$. The reaction mixture was refluxed for $20 \mathrm{~h}$. The solvent was then removed and the residue was re-dissolved with dichloromethane $\left(\mathrm{CH}_{2} \mathrm{Cl}_{2} ; 30 \mathrm{ml}\right)$. The solution was washed with saturated $\mathrm{NaCl}$. After removing the $\mathrm{CH}_{2} \mathrm{Cl}_{2}$, the residue was purified by chromatography to give compound 3 (0.66 g, 48\%) as colorless oil. HRMS: $\left(\mathrm{ESI}^{+}\right)$calculated for $\mathrm{C}_{43} \mathrm{H}_{78} \mathrm{O}_{9} \mathrm{Na}[\mathrm{M}+\mathrm{Na}]^{+}, 761.5544$; found, 761.5540. Elemental analysis: Calculated, C, 69.88; H, 10.64; found, C, 69.76; H, 10.79 .

Synthesis of FA-modified ligand (compound 4). To a solution of FA $(0.50 \mathrm{~g}, 1.13 \mathrm{mmol})$ and compound $3(0.42 \mathrm{~g}$, $0.57 \mathrm{mmol}$ ) in dimethyl sulfoxide (DMSO; $10 \mathrm{ml}$ ) was added 1-(3-dimethylaminopropyl)-3-ethylcarbodiimide hydrochloride (EDC; $0.11 \mathrm{~g}, 0.57 \mathrm{mmol}$ ) and N-hydroxysuccinimide (NHS; $65 \mathrm{mg}, 0.57 \mathrm{mmol}$ ). The resulting mixture was stirred at room temperature for $24 \mathrm{~h}$. The by-product was removed by filtration. The product was precipitated from the filtrate by the addition of diethyl ether.
A yellow solid was obtained, which was purified by flash chromatography to yield the FA-modified ligand (compound 4; 0.20 g, 30\%). HRMS: $\left(\mathrm{ESI}^{+}\right)$calculated for $\mathrm{C}_{62} \mathrm{H}_{95} \mathrm{~N}_{7} \mathrm{O}_{14} \mathrm{Na}$ $[\mathrm{M}+\mathrm{Na}]^{+}, 1,161.6937$; found, 1,161.6930. Elemental analysis: Calculated, C, 64.06; H, 8.24; N, 8.43; found C, 64.18; H, 8.33; N, 8.29.

Preparation and characterization of liposomes. Lidocaine-loaded liposomes were prepared using the thin-film hydration method $(20,21)$. The ratio of the components was optimized as follows: i) Conventional liposome (Lip), soybean phosphatidylcholine (SPC; Shanghai Taiwei Pharmaceutical Co., Ltd.)/cholesterol in a molar ratio of 62:33; ii) FA-modified liposome (FA-Lip), SPC/cholesterol/compound 4 in a molar ratio of 62:33:3. In brief, all the lipid materials were dissolved in a mixed solvent comprising $\mathrm{CH}_{2} \mathrm{Cl}_{2}$ and methanol (2:1 v/v), and the solution was then warmed on a rotary evaporator at $37^{\circ} \mathrm{C}$ to remove the solvent and form a thin film. After drying in a vacuum for $10 \mathrm{~h}$, the film was hydrated with PBS (pH 7.4) at $20^{\circ} \mathrm{C}$ for $30 \mathrm{~min}$ and sonicated intermittently at $80 \mathrm{~W}$ for $80 \mathrm{sec}$ to obtain the liposomes. An appropriate amount of lidocaine (weight ratio of procaine/lipid, 1/30) or 1,2-dioleoyl-sn-glycero-3-phosphoethanolamine-Ncarboxyfluorescein (CFPE; final concentration, $20 \mu \mathrm{g} / \mathrm{ml}$ ) was added to the solution of lipid before removing the solvent to prepare lidocaine-loaded liposomes (Lid-Lip and Lid-FA-Lip) or CFPE-labeled liposomes (CFPE-Lip and CFPE-FA-Lip).

The entrapment efficiency [EE (\%)] and drug loading efficiency [DL (\%)] of lidocaine by the liposomes were determined using high performance liquid chromatography (HPLC). The detection was performed on an Agilent 1200 HPLC System with an Ultimate LP-C18 column $(4.6 \times 250 \mathrm{~mm}, 5 \mu \mathrm{m})$ (both from Agilent Technologies, Inc.) at $25^{\circ} \mathrm{C}$. The mobile phase comprised water and methanol $(70: 30 \mathrm{v} / \mathrm{v})$ with a flow rate of $1.0 \mathrm{ml} / \mathrm{min}$. A $10-\mu 1$ aliquot of the lidocaine-containing sample was injected and the detection wavelength was $210 \mathrm{~nm}$. The EE (\%) and DL (\%) were calculated according to the following equations: $\mathrm{EE}(\%)=$ weight of encapsulated lidocaine/total weight of lidocaine, and DL (\%)=weight of encapsulated lidocaine/total weight of liposome. In addition, the size and $\zeta$ potential of Lid-Lip and Lid-FA-Lip were detected using a Malvern Zeta sizer Nano ZS90 (Malvern Panalytical).

Release by Lid-Lip and Lid-FA-Lip in vitro. The release behavior of lidocaine from the Lid-Lip and Lid-FA-Lip liposomes was assessed using dialysis. Briefly, the lidocaine-loaded liposomes were put into a dialysis bag (molecular weight cut-off, 8,000-14,000 Da) with $50 \mathrm{ml}$ PBS containing $0.1 \%$ Tween $-80(\mathrm{v} / \mathrm{v})$ as release medium. The bags were shaken at $37^{\circ} \mathrm{C}$ with gentle oscillation $(45 \mathrm{rpm})$. At various time points between 0 and $48 \mathrm{~h}$, an $0.1-\mathrm{ml}$ sample was extracted and replaced with fresh medium. The release behavior from free lidocaine was also analyzed as a control. Subsequently, the amount of lidocaine was detected by the aforementioned HPLC method.

Stability of Lid-Lip and Lid-FA-Lip in vitro. The stability of the Lid-Lip and Lid-FA-Lip liposomes was determined by the measurement of turbidity variations in the presence of fetal bovine serum (FBS; Thermo Fisher Scientific, Inc.). 
This comprised mixing each liposome formulation with an equal volume of FBS, and then continuously shaking (50 rpm) at $37^{\circ} \mathrm{C}$. The transmittance was measured between 0 and $48 \mathrm{~h}$ using a microplate reader (Bio-Rad model 550; Bio-Rad Laboratories, Inc.) at a wavelength of $750 \mathrm{~nm}$. The transmittance in PBS was defined as $100 \%$.

Hemolysis assays. A female BALB/c mouse (22 g; 4 weeks old; weight, 18-22 g) was supplied by Beijing Vital River Laboratory Animal Technology Co., Ltd. The mouse was kept in a $20^{\circ} \mathrm{C}$ environment with $40-60 \%$ humidity and a 12-h cycle of day and night. Free food and water were provided during the feeding process, and clean and hygienic feeding conditions were maintained. The mouse was anesthetized by the intraperitoneal injection of pentobarbital sodium at a dose of $50 \mathrm{mg} / \mathrm{kg}$. Fresh blood $(0.1 \mathrm{ml})$ was collected from the orbit, and centrifuged $\left(4^{\circ} \mathrm{C}\right)$ at $2,000 \times \mathrm{g}$ for $5 \mathrm{~min}$. The supernatant was discarded and the precipitated red blood cells were washed with PBS until the supernatant was colorless. The cells were then re-suspended in PBS to a concentration of $2 \%(\mathrm{w} / \mathrm{v})$. Afterwards, Lid-Lip and Lid-FA-Lip were diluted with PBS to provide liposome samples with lipid concentrations of 10, 25, 50, 100, 200 and $400 \mathrm{nM}$. Subsequently, each liposome sample $(0.4 \mathrm{ml})$ was mixed with red blood cell suspension $(0.1 \mathrm{ml})$ and the mixture was incubated at $37^{\circ} \mathrm{C}$ for $2 \mathrm{~h}$ with gentle shaking $(50 \mathrm{rpm})$. After centrifuging $\left(4^{\circ} \mathrm{C}\right)$ at $6,000 \mathrm{x}$ g for $10 \mathrm{~min}$, the absorbance of the supernatant at $540 \mathrm{~nm}\left(\mathrm{~A}_{540}\right)$ was measured using a microplate reader. The hemolysis rate of each sample was calculated as follows: Hemolysis $(\%)=\left(\mathrm{A}_{540}\right.$ sample- $\mathrm{A}_{540}$ negative $) /\left(\mathrm{A}_{540}\right.$ positive- $\mathrm{A}_{540}$ negative $) \mathrm{x} 100$. The absorbances of PBS and Triton X-100 mixed with cell solution were defined as 0 and $100 \%$, respectively.

Cell culture. Brain capillary endothelial cells (BCECs) and the U87 glioblastoma of unknown origin cell line were obtained from The Cell Bank of Type Culture Collection of the Chinese Academy of Sciences. The cells were cultured in Dulbecco's modified Eagle's medium (DMEM; Gibco; Thermo Fisher Scientific, Inc.) supplemented with 10\% FBS and $1 \%$ penicillin/streptomycin (HyClone; Cytiva).

MTT assay. Cytotoxicity was analyzed using a standard MTT-based colorimetric assay. In brief, BCECs and U87 cells were cultured in DMEM supplemented with $10 \% \mathrm{FBS}$ at $37^{\circ} \mathrm{C}$ in a humidified incubator containing $5 \% \mathrm{CO}_{2}$. The cells were seeded into 96 -well plates at a density of $\sim 5 \times 10^{3}$ cells/well and cultured for $24 \mathrm{~h}$. Fresh medium containing lidocaine, Lid-Lip or Lid-FA-Lip was applied to the cells with lidocaine concentrations ranging from $0.01-10 \mathrm{mM}$. The cells were incubated for another $24 \mathrm{~h}$ at $37^{\circ} \mathrm{C}$, and then MTT $(5 \mathrm{mg} / \mathrm{ml})$ was added to each well with further culturing for another $4 \mathrm{~h}$ at $37^{\circ} \mathrm{C}$. After this, the cells were lysed using $200 \mu \mathrm{l}$ DMSO and the absorbance at $490 \mathrm{~nm}\left(\mathrm{~A}_{490}\right)$ was measured using a microplate reader. The percentages of surviving cells were calculated using the following equation: Survival $(\%)=\left[\left(\mathrm{A}_{490}\right.\right.$ treated cells $) /\left(\mathrm{A}_{490}\right.$ control cells $\left.)\right] \times 100$.

Transendothelial migration in a BBB model. Millicell Hanging Cell Culture Inserts (Corning, Inc.) were used to build an in vitro $\mathrm{BBB}$ model as previously described (22). In brief,
BCECs were maintained in DMEM with 10\% FBS (Gibco; Thermo Fisher Scientific, Inc.) and seeded on 6-well plates at a density of $1 \times 10^{6}$ cells/well and incubated for 7 days at $37^{\circ} \mathrm{C}$. The transendothelial electric resistance (TEER) of the BBB model was measured using a Millicell ERS volt-ohm meter (EMD Millipore). Only BCEC monolayers with TEER values $>200 \Omega$ were used for further experiments. Additionally, the U87 cells were plated on another 6 -well plate ( $1 \times 10^{6}$ cells/well). The cell culture inserts with BCEC monolayers were transferred to the plates containing the U87 cells and the cells were co-cultured for another $24 \mathrm{~h}$. Subsequently, the CFPE-labeled liposomes (CFPE-Lip and CFPE-FA-Lip; final concentration of CFPE, $2 \mu \mathrm{g} / \mathrm{ml}$ ) were added to the cell culture inserts (donor chamber) of the BBB model and incubated at $37^{\circ} \mathrm{C}$ for $4 \mathrm{~h}$. The BCECs and U87 cells were then each washed with cold PBS three times, trypsinized and finally resuspended in $0.5 \mathrm{ml}$ PBS. The fluorescent intensity of the two types of cells was measured using a flow cytometer (Cytomics FC500; Beckman Coulter, Inc.) and the flow cytometer's integral analysis software (FlowJo 10; Becton, Dickinson and Company).

Wound healing assays. A wound healing assay was performed to evaluate the effects of the liposomal formulations on cell migration. U87 cells at a confluence of $100 \%$ and treated with Lid-Lip or Lid-FA-Lip ( $1 \mathrm{mM}$ lidocaine; $37^{\circ} \mathrm{C} ; 24 \mathrm{~h}$ ) were wounded by scraping with a $10-\mu 1$ pipette tip and washed twice. The cells were then cultured with serum-free DMEM to stimulate wound healing. Photographic images were captured by an Olympus light microscope before culture and at the 24-h time point to evaluate the migration of the U87 cells. The images were then analyzed using ImageJ 8.0 software (National Institutes of Health).

Cell apoptosis assays. Cell apoptosis was evaluated using Annexin V-FITC and propidium iodide (PI) staining (Sangon Biotech Co., Ltd.). U87 cells were treated with Lid-Lip or Lid-FA-Lip for $48 \mathrm{~h}\left(0-10 \mathrm{mM}\right.$ lidocaine; $\left.37^{\circ} \mathrm{C}\right)$, then centrifuged $(200 \mathrm{x} \mathrm{g})$ at $4^{\circ} \mathrm{C}$ for $3 \mathrm{~min}$ and washed with PBS. Subsequently, the cells were re-suspended in $100 \mu \mathrm{l}$ binding buffer with $5 \mu \mathrm{l}$ Annexin V-FITC and incubated at room temperature for $10 \mathrm{~min}$. Then, $5 \mu \mathrm{l}$ PI solution was added and the cells were incubated for another $5 \mathrm{~min}$ at room temperature. The proportion of apoptotic cells was analyzed using a flow cytometer (Cytomics FC500; Beckman Coulter, Inc.) and the flow cytometer's integral analysis software.

Immunoblot assay. Following treatment of U87 cells with Lid-FA-Lip ( $1 \mathrm{mM}$ lidocaine, $37^{\circ} \mathrm{C}$ for $24 \mathrm{~h}$ ), total protein was extracted with RIPA buffer (Beyotime Institute of Biotechnology) and protein determination was performed using the BCA method. Protein samples (10 $\mu \mathrm{g}$ loaded per lane) were separated on $10 \%$ SDS-PAGE and transferred onto PVDF membranes (250 mA; $2 \mathrm{~h})$, which were then blocked with $5 \%$ milk in TBS with $0.05 \%$ Tween- 20 at $25^{\circ} \mathrm{C}$ for $2 \mathrm{~h}$. The PVDF membranes were subsequently treated with primary antibodies targeting the following proteins (all from Abcam): Bcl-2 (ab32124; dilution, 1:500), matrix metalloproteinase 2 (MMP2; ab92536; dilution, 1:500), Ki67 (ab92742; dilution, 1:1,000), phosphorylated (p-)PI3Kp85 (phospho Y607; ab182651; dilution, 1:1,000), PI3Kp85 (ab135253; dilution, 

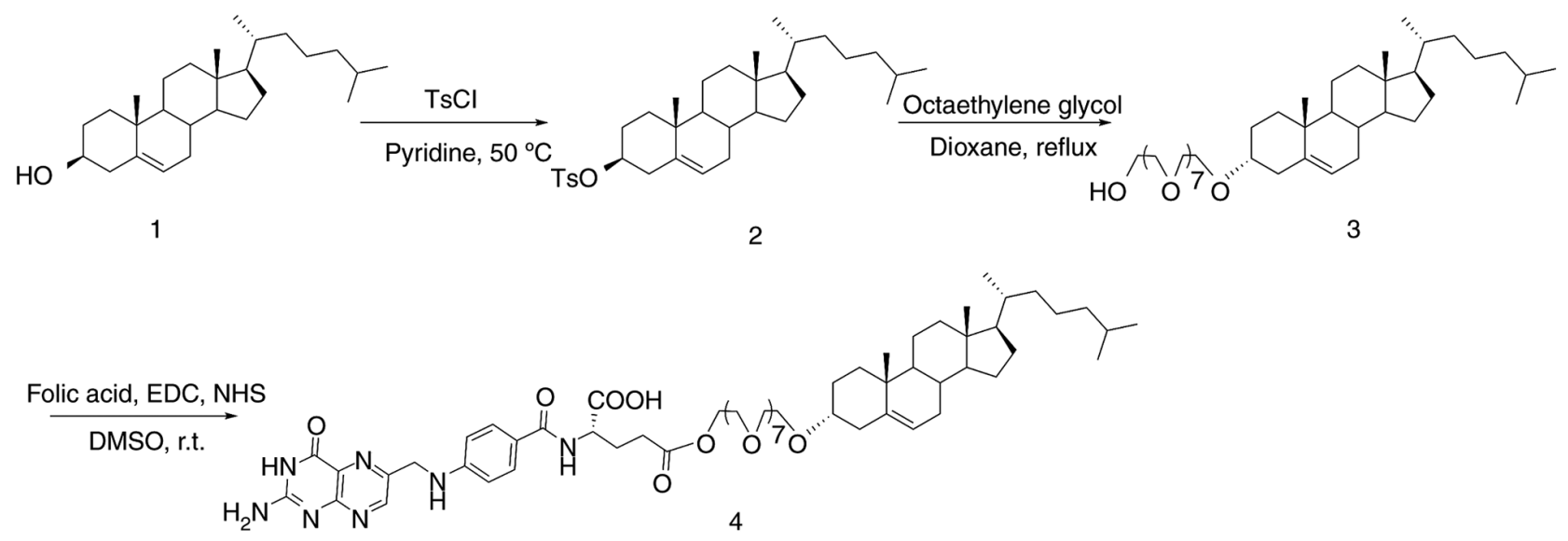

Figure 1. Synthesis of FA-modified ligand. Compound 1 (cholesterol) was esterified with $\mathrm{TsCl}$ in pyridine to yield compound 2, followed by etherification reaction with octaethylene glycol to obtain compound 3. The esterification of compound 3 with folic acid in the presence of EDC and NHS produced ligand 4. FA, folic acid; EDC, 1-(3-dimethylaminopropyl)-3-ethylcarbodiimide hydrochloride; NHS, N-hydroxysuccinimide; r.t., room temperature.

1:500), p-AKT (phospho T308; ab38449; dilution, 1:1,000), AKT (ab18785; dilution, 1:1,000) and GAPDH (ab8245; dilution, $1: 3,000)$ at room temperature for $2 \mathrm{~h}$. The membranes were then incubated with anti-rabbit (cat. no. ab6271; Abcam) or anti-mouse (cat. no. ab6728; Abcam) HRP-conjugated secondary antibodies (1:5,000 dilution) at room temperature for $1 \mathrm{~h}$. Signals were then visualized using an ECL kit (Novex $^{\mathrm{TM}}$ ECL Chemiluminescent Substrate Reagent kit; Thermo Fisher Scientific, Inc.) and visualized by ImageJ 8.0 software (National Institutes of Health).

Antitumor activity in vivo. All experiments, including the extraction of blood from a mouse for the hemolysis assay, were approved by the Ethics Committee of the Second Hospital of Tianjin Medical University. A total of 18 female BALB/c nude mice (4 weeks old; weight, 18-22 g) were supplied by Beijing Vital River Laboratory Animal Technology Co., Ltd. The mice were provided with food and water ad libitum, and were kept under specific pathogen-free conditions at $20^{\circ} \mathrm{C}$ and $60 \%$ humidity with a 12 -h alternating light/dark cycle. Adequate humanitarian care was given, and no mice died during the experiment. For the tumor growth assay, $\sim 5 \times 10^{5}$ U87 cells were mixed with Matrigel (BD Biosciences) at a ratio of 2:1, and subcutaneously implanted into the abdominal region of the mice for tumor induction over 7 days. To detect the in vivo antitumor effects of treatment on the glioma xenografts, the mice were divided into three groups ( $n=6 /$ group). Lidocaine, Lid-Lip and Lid-FA-Lip were administered at a dose of $10 \mathrm{mg} / \mathrm{kg}$ (calculated as lidocaine) via intravenous injection every 3 days. After another 12 days, mice were sacrificed by cervical dislocation and the lack of heartbeat was confirmed to validate death. Three mice from each group were sacrificed at the 7-day time point and after 12 days of treatment. The tumors were then isolated from the mice and the weights of the tumors were measured. Tumor volume was also calculated as follows: Tumor volume $\left(\mathrm{mm}^{3}\right)=$ tumor length $(\mathrm{mm}) \mathrm{x}$ tumor width $(\mathrm{mm})^{2} / 2$.

Statistical analysis. All data are presented as the mean \pm standard deviation. The statistical analyses were conducted using GraphPad Prism 6.0 (GraphPad Software, Inc.). Unpaired Student's t-test was used for statistical comparisons between two groups. Statistical comparisons among multiple groups were performed via one-way analysis of variance followed by Tukey's post hoc tests. $\mathrm{P}<0.05$ was considered to indicate a statistically significant difference.

\section{Results}

Chemistry. The synthetic pathway of the FA-modified ligand used in the present study is outlined in Fig. 1. Briefly, cholesterol was esterified with $p$-toluenesulfonyl chloride in pyridine to yield cholesteryl tosylate as an intermediate, followed by etherification with octaethylene glycol to obtain octaethylene glycol monocholesteryl ether. Subsequent esterification with FA in the presence of EDC and NHS yielded the FA-modified ligand.

Characterization of liposomes. For lidocaine-loaded liposomes to cross the BBB and target glioma, they should have an appropriate size and uniform distribution. The mean diameters and polydispersity index (PDI) of the liposomes prepared in the current study were detected using dynamic light scattering, and the results indicated that these liposomes had a suitable size $(\sim 110 \mathrm{~nm})$ and PDI $(<0.2)$. Furthermore, the data presented in Table I also show that the EE (\%) of the two liposomes was $>88 \%$ and the DL $(\%)$ was $\sim 2.8 \%$. Additionally, the weak negative $\zeta$ potential of Lid-FA-Lip suggests that its absorption by the reticuloendothelial system and effect on the immune response should be minimal (18).

The release behavior study of the liposomes was investigated in vitro. The results revealed that the free lidocaine exhibited a fast-release characteristic, with nearly $80 \%$ of the lidocaine being released in $10 \mathrm{~h}$ (Fig. 2A). By contrast, the lidocaine-loaded liposomes released $\sim 40 \%$ of the loaded lidocaine within $10 \mathrm{~h}$, and the release rate slowed subsequently. Fig. 2A also demonstrates that neither of the two liposome formulations exhibited an initial burst release profile.

The stability of liposomes under physiological conditions is important for their in vivo application. Therefore, the transmittances of the Lid-Lip and Lid-FA-Lip liposome formulations were evaluated over a 48 -h time period. The transmittance values remained at $>92 \%$, with had no marked differences between 
Table I. Characterization of Lid-Lip and Lid-FA-Lip (n=3).

\begin{tabular}{lccccc}
\hline Liposomes & Size $(\mathrm{nm})$ & PDI & EE $(\%)$ & DL $(\%)$ & $\zeta$ potential $(\mathrm{mV})$ \\
\hline Lid-Lip & $108.97 \pm 5.6$ & $0.189 \pm 0.043$ & $88.51 \pm 4.03$ & $2.75 \pm 0.17$ & $-5.31 \pm 0.28$ \\
Lid-FA-Lip & $112.35 \pm 9.4$ & $0.196 \pm 0.069$ & $89.76 \pm 6.98$ & $2.83 \pm 0.49$ & $-8.65 \pm 0.77$ \\
\hline
\end{tabular}

Lid-Lip, conventional lidocaine-carrying liposome; Lid-FA-Lip, FA-modified lidocaine-carrying liposome; PDI, polydispersity index; EE, entrapment efficiency; DL, drug loading efficiency.
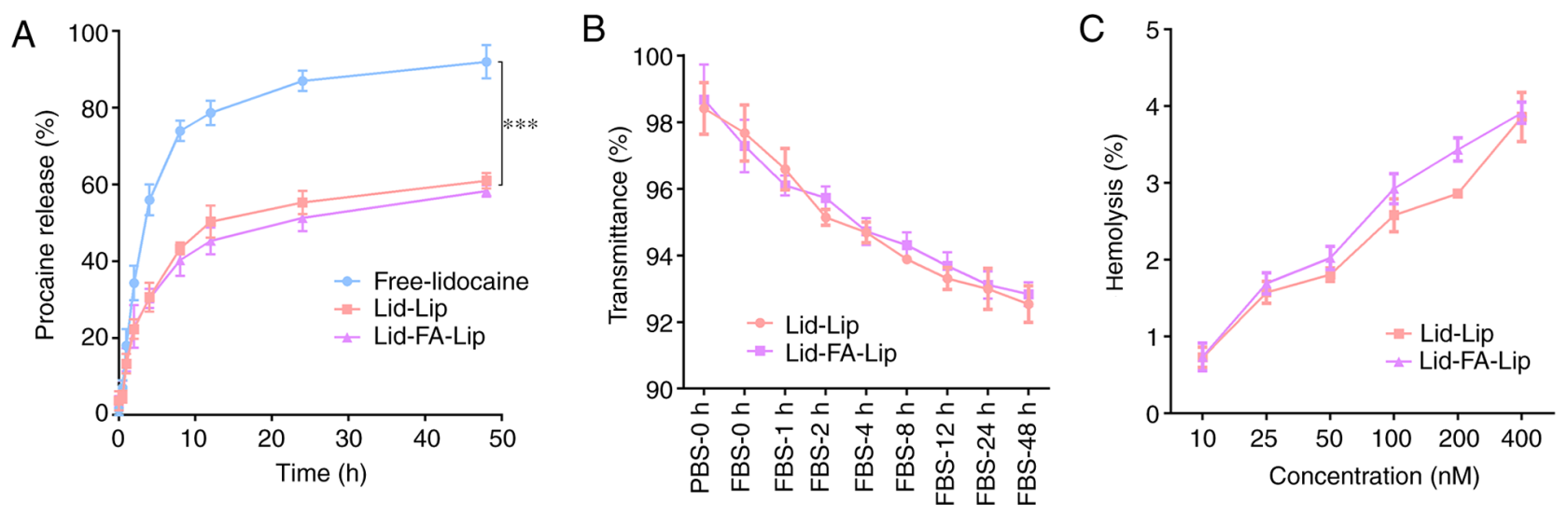

Figure 2. Characterization of the liposomes in vitro. (A) Lidocaine release profiles of free lidocaine, Lid-Lip and Lid-FA-Lip in PBS buffer (pH 7.4) containing $0.1 \%$ Tween-80 over 48 h. (B) Variations in the transmittance of Lid-Lip and Lid-FA-Lip in 50\% FBS. (C) Hemolysis percentage for Lid-Lip and Lid-FA-Lip. Results are presented as the mean \pm standard deviation $(n=3) .{ }^{* * *} \mathrm{P}<0.001$. Lid-Lip, conventional liposome carrying lidocaine; Lid-FA-Lip, FA-modified liposome carrying lidocaine. FA, folic acid; FBS, fetal bovine serum.
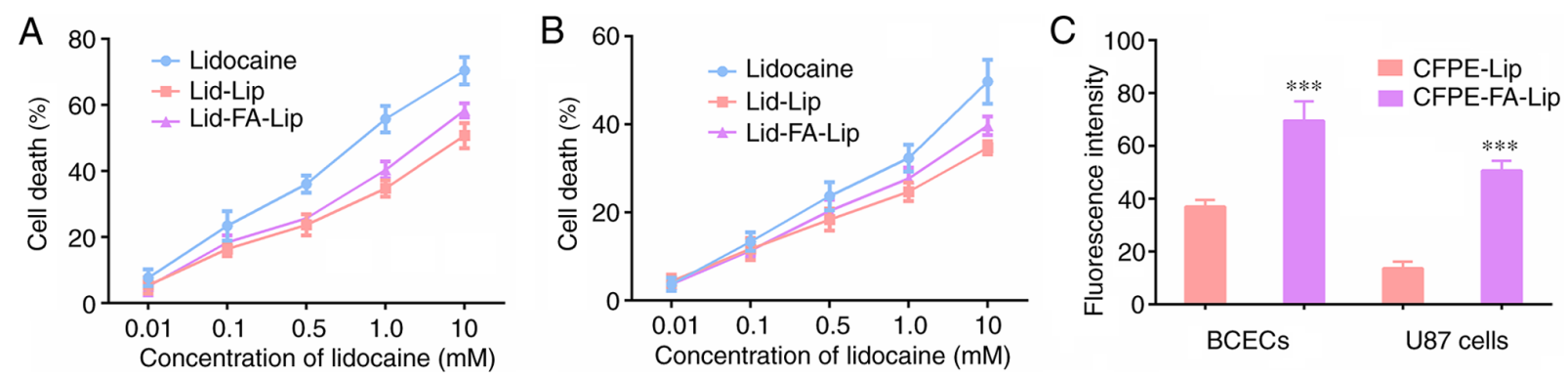

Figure 3. Lidocaine liposome modified with FA significantly suppresses the proliferation of glioma cells. Cytotoxicity of lidocaine formulations in (A) U87 glioma cells and (B) BCECs. (C) Uptake of CFPE-labeled liposomes by BCECs and U87 cells in an in vitro blood-brain barrier model. ${ }^{* * *} \mathrm{P}<0.001$. Results are presented as the mean \pm standard deviation $(\mathrm{n}=3)$. FA, folic acid; BCECs, brain capillary endothelial cells; Lid-Lip, conventional lidocaine-carrying liposome; Lid-FA-Lip, FA-modified lidocaine-carrying liposome; CFPE-Lip, conventional CFPE-carrying liposome; CFPE-FA-Lip, FA-modified CFPE-carrying liposome; CFPE, 1,2-dioleoyl-sn-glycero-3-phosphoethanolamine-N-carboxyfluorescein.

the formulations during the 48-h culture upon FBS treatment (Fig. 2B). The results suggest that the stability of Lid-Lip and Lid-FA-Lip is adequate, and supports further investigation in vivo. The hemocompatibility of Lid-Lip and Lid-FA-Lip was evaluated by hemolysis assays, and the results showed that the two types of liposome induced no marked increase in the release of hemoglobin when the phospholipid concentrations were raised to $400 \mathrm{nM}$ (Fig. 2C), which implies good biosafety.

MTT assay. The cytotoxicity of different liposome formulations to BCECs and U87 cells were evaluated using an MTT assay. As shown in Fig. 3A, free lidocaine showed a higher cytotoxic effect than lidocaine-loaded liposomes. This is likely because the free drug was transported into the cells directly by passive diffusion, while the liposomes had to undergo a drug-release process. In addition,Lid-FA-Lip exhibited a slightly stronger antiproliferative effect than Lid-Lip, which may be due to the modification by FA enhancing cellular uptake. The inhibitory effect of the lidocaine formulations on BCEC growth was also evaluated (Fig. 3B), and the results demonstrated that the formulations had reduced cytotoxic effects on the survival of endothelial cells, which indicated the antitumor specificity of lidocaine.

Transendothelial migration in a BBB model. BCECs were used to establish a BBB model in vitro. BCECs on the Transwell membrane from the donor chamber and U87 cells from the 
A

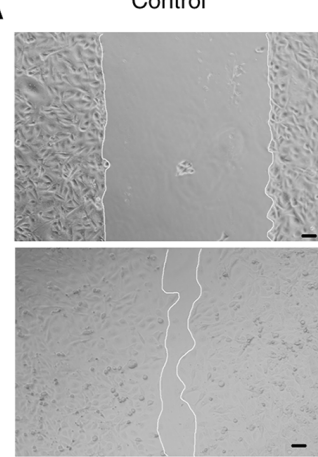

C

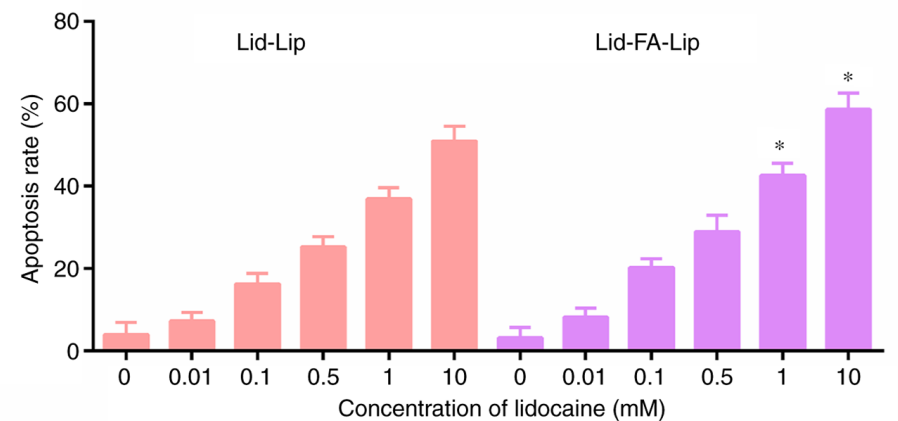

$\mathrm{E}$

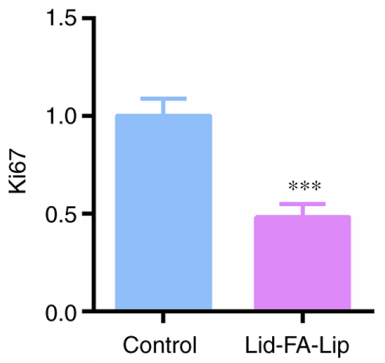

Lid-Lip

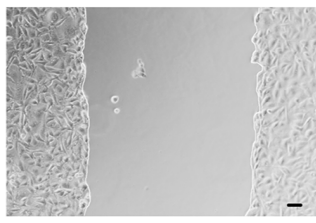

$\mathrm{F}$

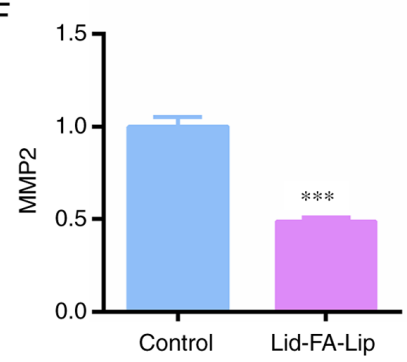

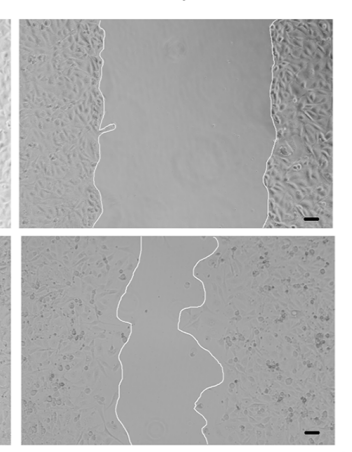

D

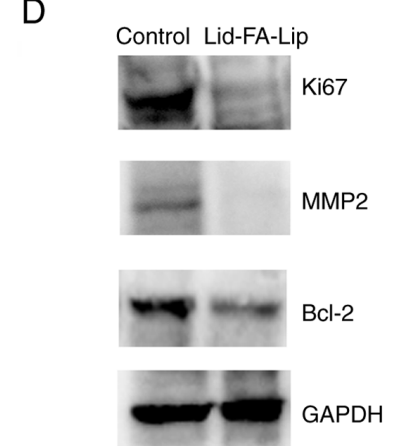

B

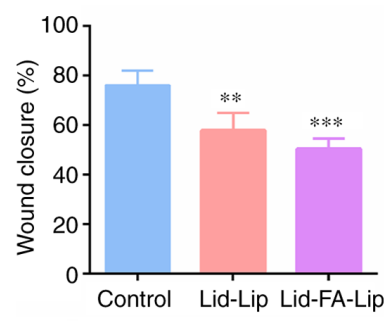

G

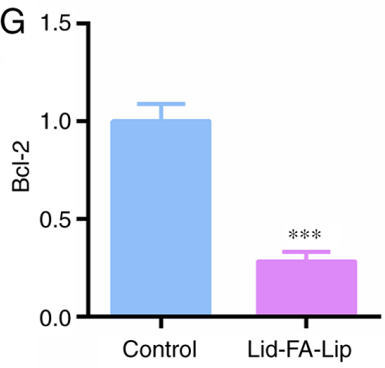

Figure 4. Lidocaine liposome modified with FA inhibits the migration of glioma cells and stimulates cell apoptosis. (A) Wound closure assay was performed to evaluate the migration of U87 cells after treatment with lidocaine-loaded liposomes. Scale bar, $5 \mu \mathrm{m}$. (B) Quantitative analysis of the wound closure assay. (C) Flow cytometry assays of Annexin V-FITC and PI staining were performed to evaluate the apoptosis of U87 cells, and a comparison of quantified apoptosis rates among different groups is shown. (D) Representative immunoblots showing the expression of Ki67, MMP2 and Bcl-2 in U87 cells following treatment with Lid-FA-Lip. (E-G) Results for the quantitative analysis of (E) Ki67, (F) MMP2 and (G) Bcl-2 expression by western blotting. Results are presented as the mean \pm standard deviation $(\mathrm{n}=3)$. ${ }^{*} \mathrm{P}<0.05,{ }^{* *} \mathrm{P}<0.01,{ }^{* * * *} \mathrm{P}<0.001$. FA, folic acid; Lid-Lip, conventional lidocaine-carrying liposome; Lid-FA-Lip, FA-modified lidocaine-carrying liposome; MMP2, matrix metalloproteinase 2.

acceptor chamber were collected, and the fluorescence intensity was measured using a flow cytometer. The results indicated that the BCEC monolayer on the Transwell membrane took up and internalized the liposomes (Figs. 3C and S1). In addition, the fluorescence intensity of the U87 cells in the model in which the BCECs were treated with the CFPE-FA-Lip formulation was significantly increased compared with that in which the BCECs were treated with the CFPE-Lip formulation, which indicates that after penetrating the BCEC monolayer, CFPE-FA-Lip was transported across the BCEC monolayer and delivered into the U87 cells more effectively than CFPE-Lip. These results suggest that the transport of liposomes across the simulated $\mathrm{BBB}$ was enhanced when they were modified with FA.

Wound closure assay. A wound closure assay was performed to investigate the effects of Lid-Lip and Lid-FA-Lip on U87 cell migration. The results reveal that Lid-FA-Lip significantly suppressed the migration of U87 cells compared with that of the control cells (Fig. 4A and B), and indicate the strong suppressive effect of Lid-FA-Lip on glioma cell motility.

Cell apoptosis detection assays. Subsequently, the effects of Lid-Lip and Lid-FA-Lip on the apoptosis of U87 cells were analyzed using flow cytometry. Notably, the results demonstrated that Lid-FA-Lip stimulated cell apoptosis in a concentration-dependent manner (Figs. 4C and S2) and can significantly stimulate U87 cell apoptosis.

Western blot analyses demonstrated that Lid-FA-Lip significantly decreased the expression levels of Ki67, a cell proliferation marker (Fig. 4D and E), MMP2, a cell migration marker (Fig. 4D and F) and Bcl-2, a cell apoptosis marker (Fig. 4D and G) compared with those in control cells.

Lid-FA-Lip exhibits antitumor activity in glioma by targeting the PIBK/AKT pathway. The aforementioned results indicate 
A

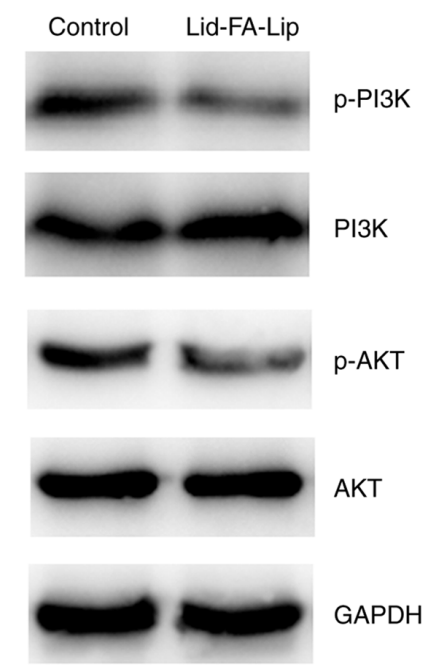

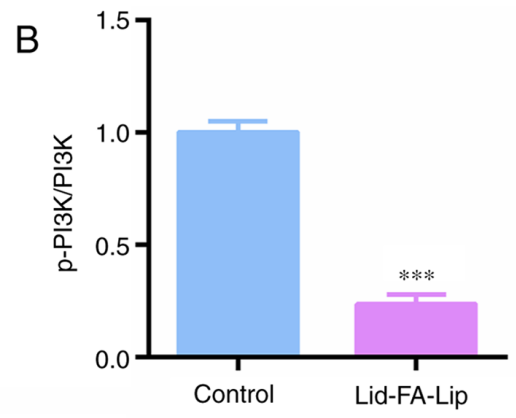

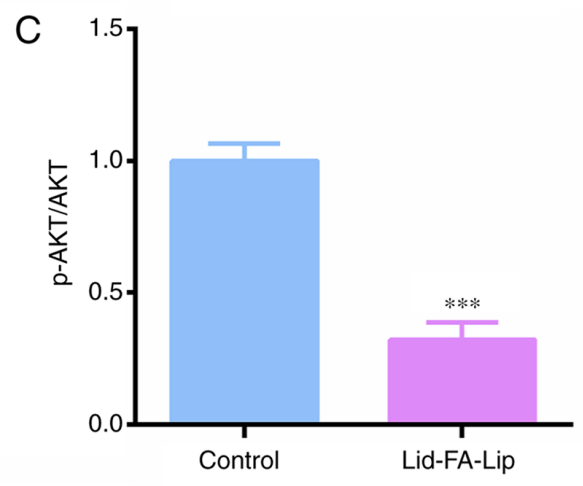

$\mathrm{E}$
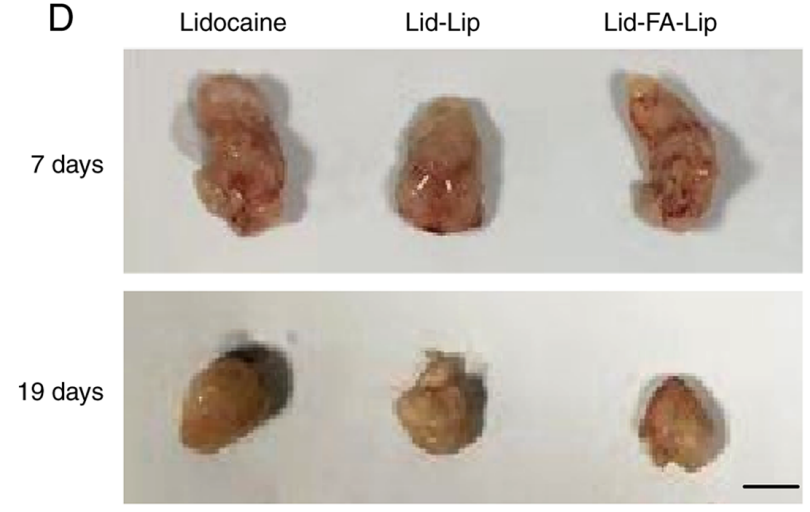

$\mathrm{F}$

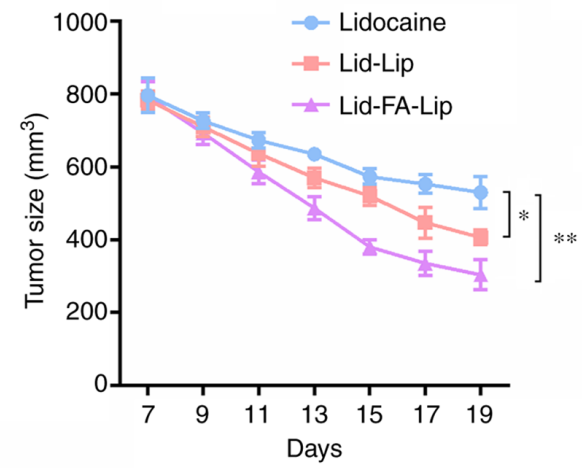

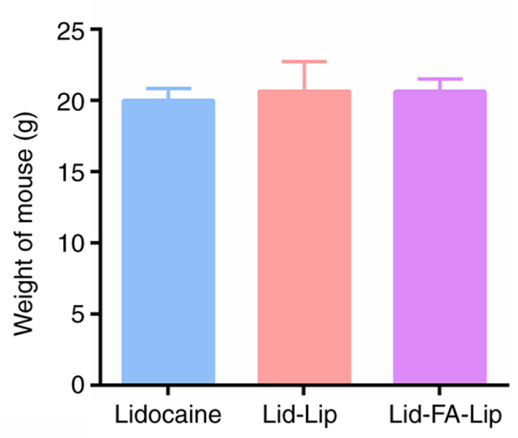

G

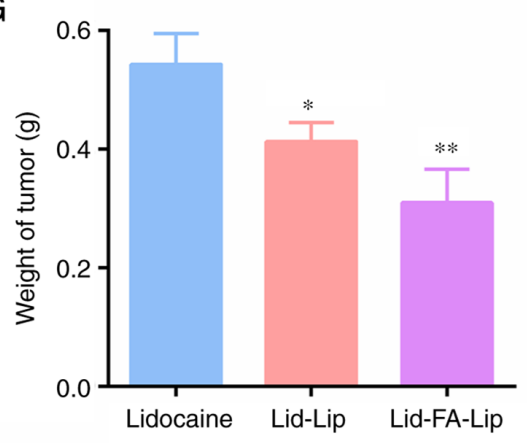

Figure 5. Lidocaine liposome modified with FA inhibits the tumor growth of glioma cells via the PI3K/AKT pathway. (A) Representative immunoblots showing p-PI3K, PI3K, p-AKT and AKT levels in U87 cells following treatment with Lid-FA-Lip. Quantitative analysis of the (B) p-PI3K/PI3K and (C) p-AKT/AKT ratios. (D) Representative photographs of tumors formed by U87 cells, isolated from nude mice following the treatment with different lidocaine formulations ( $\mathrm{n}=6 /$ group). The upper images are the photographs of the tumors at 7 days, and the lower images show the tumors at 19 days. Scale bar, $5 \mathrm{~mm}$. (E) Weights of the mice were measured. (F) Tumor growth curves for the control and the liposomal treatment groups are shown. (G) Tumor weights were measured and compared. Results are presented as the mean \pm standard deviation. ${ }^{*} \mathrm{P}<0.05,{ }^{* *} \mathrm{P}<0.01,{ }^{* * * *} \mathrm{P}<0.001$. FA, folic acid; Lid-Lip, conventional lidocaine-carrying liposome; Lid-FA-Lip, FA-modified lidocaine-carrying liposome; p-, phosphorylated.

that Lid-FA-Lip is able to suppress the proliferation and migration of glioma cells and stimulate cell apoptosis in vitro. The PI3K/AKT pathway is known as a target of lidocaine (23); therefore, the effects of Lid-FA-Lip on this pathway were evaluated. Western blot assays demonstrated that the phosphorylation levels of PI3Kp85 were significantly decreased following treatment with Lid-FA-Lip compared with the control (Fig. 5A and B). Additionally, Lid-FA-Lip treatment 
significantly decreased AKT phosphorylation levels compared with those of the untreated control (Fig. 5A and C). These results suggest that the antitumor activity of Lid-FA-Lip in glioma is mediated via targeting the PI3K/AKT pathway.

The antitumor activity of Lid-FA-Lip was also validated in vivo. The findings of the xenograft experiment confirm the effectiveness of this type of targeted therapy. Smaller tumors were observed in mice from the Lid-FA-Lip treatment group compared with the Lid-Lip and control groups. Representative images of tumors from each group are shown in Fig. 5D. The results confirm that, although there was no significant change in the weight of the mice (Fig. 5E), the tumor volume (Fig. 5F) and tumor weight (Fig. 5G) of the mice treated with Lid-FA-Lip was significantly reduced. This demonstrates the antitumor effects of Lid-FA-Lip.

\section{Discussion}

Glioma is a craniocerebral malignancy affecting glial cells (1), and accounts for $\sim 60 \%$ of all intracranial tumors (24). Although the BBB results in glioma having an extremely low rate of metastasis it also hinders the treatment of glioma using therapeutic drugs. Therefore, the development of novel targeted drug delivery systems for glioma is necessary.

Lidocaine is widely used as a local anesthetic. In addition to having anesthetic effects, lidocaine has also been reported to have inhibitory effects on tumors (25-27). A previous study demonstrated that lidocaine can inhibit vascular endothelial growth factor-A-induced angiogenesis, and therefore may be able to suppress cancer progression (28). Additionally, lidocaine has been reported to inhibit the proliferation and invasion of hepatocellular carcinoma cells by inhibiting the PI3K/Akt pathway (23). Similarly, another study demonstrated that the inhibitory effect of lidocaine on lung cancer cell proliferation is mediated via PI3K/AKT and EGFR pathways (29). These studies, together with the findings of the present study, indicate the potential role of lidocaine in tumor therapy.

In the present study, an FA-cholesterol derivative was designed and synthesized, which was characterized by HMRS and elemental analysis to confirm its structure. A lidocaine-loaded liposomal drug delivery system modified with this ligand was prepared. Analysis of the characteristics of the liposome in vitro suggested that it had a suitable size and PDI, which should contribute to the achievement of passive targeting via enhanced permeability and retention effects. In addition, the slow release behavior exhibited in the drug release assay demonstrates that Lid-FA-Lip significantly improved the release behavior of lidocaine with a sustained release effect. The hemolysis rate was $<5 \%$, which is regarded as nontoxic (20). The results of all the characterization assays indicated that the liposomes were suitable for evaluation in further in vivo and in vitro experiments.

The present study demonstrated that Lid-FA-Lip had greater ability to penetrate the BBB in a cell model and improve cellular uptake compared with Lid-Lip, suggesting that the transport capacity of the liposomes was enhanced by modification with FA. The results also revealed that Lid-FA-Lip was able to inhibit cell migration and induce cell apoptosis. Using wound closure assays, it was observed that Lid-FA-Lip suppressed the wound healing of glioma cells,
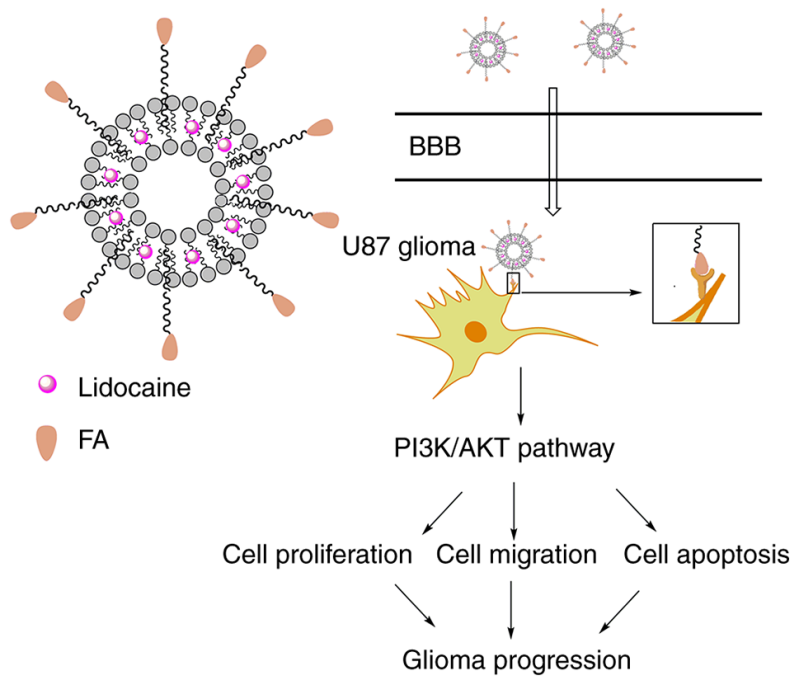

Figure 6. Schematic illustration of Lid-FA-Lip crossing the BBB and targeting glioma cells. FA is an effective ligand for crossing the BBB and glioma treatment, and Lid-FA-Lip exhibited antitumor effects via targeting the PI3K/AKT pathway. Lid-FA-Lip, FA-modified lidocaine-carrying liposome; BBB, blood-brain barrier; FA, folic acid.

with a reduction in MMP2 expression compared with that in the control group. Though flow cytometry and western blotting assays, it was noted that Lid-FA-Lip treatment significantly stimulated the apoptosis of glioma cells, with a reduction in the expression levels of $\mathrm{Bcl}-2$, an anti-apoptotic biomarker. Tumor angiogenesis can affect the prognosis of patients with tumors. Endothelial cells are important in the formation of new blood vessels that feed the tumor with nutrients. Notably, the present confirmed that Lid-FA-Lip had superior antitumor effects mediated via targeting the PI3K/AKT pathway and thereby suppressed tumor growth. The scheme in Fig. 6 illustrates the suggested mechanism. The PI3K/AKT pathway affects multiple cellular processes, including cell proliferation, migration, invasion and apoptosis $(30,31)$, and is involved in the progression of various cancers (32). A number of proteins promote the progression and metastasis of cancers, including lung cancer and pancreatic cancer, via the PI3K/AKT pathway, and drugs targeting the PI3K/AKT pathway have been used in the clinic or in clinical trials (33). The PI3K/AKT axis is also widely known to be involved the regulation of glioma progression (29). This pathway affects the proliferation, migration and apoptosis of glioma cells (29), and the results of the present study indicate that Lid-FA-Lip exerts antitumor effects via this pathway; Lid-FA-Lip reduced PI3K and AKT phosphorylation levels and therefore inhibits this pathway.

In summary, the present study aimed to develop an effective drug delivery system for the targeting of glioma and to explore the antitumor activity and mechanism of lidocaine delivered using this system. A novel FA-cholesterol derivative was designed and synthesized as a liposomal ligand. The results revealed that FA increased the ability of the liposome to penetrate an in vitro model of the BBB and improved cellular uptake. In addition, Lid-FA-Lip exhibited significant inhibitory effects on cell motility and induced the apoptosis of glioma cells. It was further confirmed that Lid-FA-Lip exerted antitumor effects by targeting the PI3K/AKT pathway 
and inhibited tumor growth in vitro. Therefore, it may be concluded that FA is an effective ligand for transporting lidocaine through the $\mathrm{BBB}$ and treating glioma, and the antitumor effects of Lid-FA-Lip are mediated via targeting the $\mathrm{PI} 3 \mathrm{~K} / \mathrm{AKT}$ pathway.

\section{Acknowledgements}

Not applicable.

\section{Funding}

This study was supported by the 2019 Middle-aged and Young Training Fund Project of the Anesthesiology Branch of Tianjin Medical Association (grant no. TJMZJJ-2019-03) and the Youth Fund of the Second Hospital of Tianjin Medical University (grant no. 2018ydey14).

\section{Availability of data and materials}

The datasets used and/or analyzed during the current study are available from the corresponding author on reasonable request.

\section{Authors' contributions}

DL, XY, BL and CY carried out the molecular biology experiments and drafted the manuscript. DL, XY, JS, MY and HW participated in the design of the study and performed the statistical analysis. DL, XY and YL conceived of the study, participated in its design and coordination and helped to draft the manuscript. DL and YL confirm the authenticity of all the raw data. All authors read and approved the final manuscript.

\section{Ethics approval and consent to participate}

All procedures performed in the current study were approved by the Ethics Committee of the Second Hospital of Tianjin Medical University.

\section{Patient consent for publication}

Not applicable.

\section{Competing interests}

The authors declare that they have no competing interests.

\section{References}

1. Dimou J and Kelly J: The biological and clinical basis for early referral of low grade glioma patients to a surgical neuro-oncologist. J Clin Neurosci 78: 20-29, 2020.

2. Liu M and Wang L: Prognostic significance of preoperative serum albumin, albumin-to-globulin ratio, and prognostic nutritional index for patients with glioma: A meta-analysis. Medicine (Baltimore) 99: e20927, 2020

3. Huang J, Yu J, Tu L, Huang N, Li H and Luo Y: Isocitrate dehydrogenase mutations in glioma: From basic discovery to therapeutics development. Front Oncol 9: 506, 2019.

4. Zhang X,Zhao L, Zhai G, Ji J and Liu A: Erratum: Multifunctional polyethylene glycol (PEG)-Poly (Lactic-Co-Glycolic Acid) (PLGA)-based nanoparticles loading doxorubicin and tetrahydrocurcumin for combined chemoradiotherapy of glioma. Med Sci Monit 26: e926333, 2020.
5. Finck T, Gempt J, Krieg SM, Meyer B, Zimmer C, Wiestler B, Kirschke JS and Sollmann N: Assessment of the extent of resection in surgery of high-grade glioma-evaluation of black blood sequences for intraoperative magnetic resonance imaging at 3 tesla. Cancers (Basel) 12: 1580, 2020.

6. Opoku-Darko M, Lang ST, Artindale J, Cairncross JG, Sevick RJ and Kelly JJP: Surgical management of incidentally discovered diffusely infiltrating low-grade glioma. J Neurosurg 129: 19-26, 2018.

7. Pisapia DJ: The updated World Health Organization glioma classification: Cellular and molecular origins of adult infiltrating gliomas. Arch Pathol Lab Med 141: 1633-1645, 2017.

8. de Blank P, Fouladi M and Huse JT: Molecular markers and targeted therapy in pediatric low-grade glioma. J Neurooncol 150: $5-15,2020$

9. Yan C, Wang J, Yang Y, Ma W and Chen X: Molecular biomarker-guided anti-angiogenic targeted therapy for malignant glioma. J Cell Mol Med 23: 4876-4882, 2019.

10. Li J, Chai Z, Lu J, Xie C, Ran D, Wang S, Zhou J and Lu W: $\alpha_{v} \beta_{3}$-targeted liposomal drug delivery system with attenuated immunogenicity enabled by linear pentapeptide for glioma therapy. J Control Release 322: 542-554, 2020.

11. He Y, Wu C, Duan J, Miao J, Ren H and Liu J: Anti-Glioma effect with targeting therapy using folate modified nano-micelles delivery curcumin. J Biomed Nanotechnol 16: 1-13, 2020.

12. Ye L, Zhang Y, Chen YJ and Liu Q: Anti-tumor effects of lidocaine on human gastric cancer cells in vitro. Bratisl Lek Listy 120: 212-217, 2019.

13. Li YC, Wang Y, Li DD, Zhang Y, Zhao TC and Li CF: Procaine is a specific DNA methylation inhibitor with anti-tumor effect for human gastric cancer. J Cell Biochem 119: 2440-2449, 2018.

14. Zuckerman LM, Frames WL, Mirshahidi HR, Williams NL, Shields TG, Otoukesh S and Mirshahidi S: Antiproliferative effect of bupivacaine on patient-derived sarcoma cells. Mol Clin Oncol 13: 7, 2020

15. Unami A, Shinohara Y, Ichikawa T and Baba Y: Biochemical and microarray analyses of bupivacaine-induced apoptosis. J Toxicol Sci 28: 77-94, 2003.

16. Zhao Y, Zhang L, Peng Y, Yue QM, Hai L, Guo L, Wang QT and Wu Y: GLUT,-mediated venlafaxine-thiamine disulfide system-glucose conjugates with 'lock-in' function for central nervous system delivery. Chem Biol Drug Des 91: 707-716, 2018.

17. Tsou YH, Zhang XQ, Zhu H, Syed S and Xu X: Drug Delivery to the brain across the blood-brain barrier using nanomaterials. Small 13: e1701921, 2017. Erratum in: Small 14: e1801588, 2018.

18. Yang Y, Zhao Z, Xie C and Zhao Y: Dual-targeting liposome modified by glutamic hexapeptide and folic acid for bone metastatic breast cancer. Chem Phys Lipids 228: 104882, 2020.

19. Akal ZU, Alpsoy L and Baykal A: Superparamagnetic iron oxide conjugated with folic acid and carboxylated quercetin for chemotherapy applications. Ceram Int 42: 9065-9072, 2016.

20. Zhao Z, Chen C, Xie C and Zhao Y: Design, synthesis and evaluation of liposomes modified with dendritic aspartic acid for bone-specific targeting. Chem Phys Lipids 226: 104832, 2020.

21. Zhao Z, Zhao Y, Xie C, Chen C, Lin D, Wang S, Lin D, Cui X, Guo $\mathrm{Z}$ and Zhou J: Dual-active targeting liposomes drug delivery system for bone metastatic breast cancer: Synthesis and biological evaluation. Chem Phys Lipids 223: 104785, 2019.

22. Su Z, Xing L, Chen Y, Xu Y, Yang F, Zhang C, Ping Q and Xiao Y: Lactoferrin-Modified Poly(ethylene glycol)-Grafted BSA nanoparticles as a dual-targeting carrier for treating brain gliomas. Mol Pharm 11: 1823-1834, 2014.

23. Zhang Y, Jia J, Jin W, Cao J, Fu T, Ma D and Zhang Y: Lidocaine inhibits the proliferation and invasion of hepatocellular carcinoma by downregulating USP14 induced PI3K/Akt pathway. Pathol Res Pract 216: 152963, 2020.

24. Habimana-Griffin L, Ye D, Carpenter J, Prior J, Sudlow G, Marsala L, Mixdorf M, Rubin J, Chen H and Achilefu S: Intracranial glioma xenograft model rapidly reestablishes blood-brain barrier integrity for longitudinal imaging of tumor progression using fluorescence molecular tomography and contrast agents. J Biomed Opt 25: 1-13, 2020.

25. Du J, Zhang L, Ma H, Wang Y and Wang P: Lidocaine suppresses cell proliferation and aerobic glycolysis by regulating circHOMER1/miR-138-5p/HEY1 axis in colorectal cancer. Cancer Manag Res 12: 5009-5022, 2020.

26. Siekmann W, Tina E, Von Sydow AK and Gupta A: Effect of lidocaine and ropivacaine on primary (SW480) and metastatic (SW620) colon cancer cell lines. Oncol Lett 18: 395-401, 2019. 
27. Zhou D, Wang L, Cui Q, Iftikhar R, Xia Y and Xu P: Repositioning lidocaine as an anticancer drug: The role beyond anesthesia. Front Cell Dev Biol 8: 565, 2020.

28. Suzuki S, Mori A, Fukui A, Ema Y and Nishiwaki K: Lidocaine inhibits vascular endothelial growth factor-A-induced angiogenesis. J Anesth 34: 857-864, 2020

29. Sun $H$ and Sun Y: Lidocaine inhibits proliferation and metastasis of lung cancer cell via regulation of miR-539/EGFR axis. Artif Cells Nanomed Biotechnol 47: 2866-2874, 2019.

30. Cierniak S, Koktysz R, Jesiotr M, Gasowska-Bodnar A and Bodnar L: Expression of the PI3K/AKT/mTOR pathway as a prognostic factor in patients with advanced high grade serous ovarian carcinoma treated with neoadjuvant chemotherapy. Eur J Gynaecol Oncol 40: 744-751, 2019.

31. Zuo X, Li L and Sun L: Plantamajoside inhibits hypoxia-induced migration and invasion of human cervical cancer cells through the NF- $\kappa$ B and PI3K/akt pathways. J Recept Signal Transduct Res 41: 339-348, 2021.
32. Xiao Y, Deng T and Wang D: Davanone terpenoid inhibits cisplatin-resistant acute myeloid leukemia cancer cell growth by inducing caspase-dependent apoptosis, loss of mitochondrial membrane potential, inhibition of cell migration and invasion and targeting PI3K/AKT/MAPK signalling pathway. J BUON 25: 1607-1613,2020.

33. Liu Z, Mo H, Sun L, Wang L, Chen T, Yao B, Liu R, Niu Y, Tu K, $\mathrm{Xu} \mathrm{Q}$ and Yang N: Long noncoding RNA PICSAR/miR-588/EIF6 axis regulates tumorigenesis of hepatocellular carcinoma by activating PI3K/AKT/mTOR signaling pathway. Cancer Sci 111: 4118-4128, 2020.

cc) () $(9$ This work is licensed under a Creative Commons CY NO AO Attribution-NonCommercial-NoDerivatives 4.0 International (CC BY-NC-ND 4.0) License. 